\title{
Phase separation of weakly ionized polymer gels during shrinking phase transition
}

\author{
Gang Bai and Atsushi Suzuki ${ }^{\mathrm{a})}$ \\ Department of Materials Science, Yokohama National University, 79-5 Tokiwadai, Hodogaya-ku, \\ Yokohama, 240-8501 Japan
}

(Received 3 August 1999; accepted 10 September 1999)

\begin{abstract}
We have investigated the shrinking phase transition of weakly ionized poly( $N$-isopropylacrylamide) gels prepared in a cylindrical shape with submillimeter diameter. The macroscopic conformation changes were obtained on heating processes in two different methods. One is a continuous heating process with a constant temperature drift rate, and the other is an isothermal process after a steplike temperature increase beyond the transition point. It was found that the macroscopic behavior can be characterized by several conformation changes; the phase coexistence, the grain pattern, the bubble pattern, and the opaque phase. On a continuous heating process, the phase transition can be characterized by the phase coexistence of completely collapsed and swollen states for the smaller temperature drift rates; the selected portions on the surface can start to collapse at the transition point, which develops with time and finally becomes a completely collapsed phase. For the larger temperature drift rates, the phase transition starts many places on the surface, and the whole gel with surface bubble pattern gradually shrinks with time. These different processes can be clearly observed in the latter measurements, which depend on the degree of the super-heating (quench); for the shallow quenches, the number of the completely collapsed states correspondingly increased with increasing the super-heating. For the deep quenches, the gel becomes opaque, and the transparent surface skin (collapsed phase) develops with time. The stability of the phase coexistence and the relationship with the transition velocity were qualitatively discussed in terms of the classical phase-separation model based on the nucleation and the spinodal decomposition. (C) 1999 American Institute of Physics. [S0021-9606(99)50146-3]
\end{abstract}

\section{INTRODUCTION}

Polymer gel is one of the soft condensed materials of a random two-phase system; polymer (solid) and solvent (liquid). ${ }^{1}$ It is well known that such systems, made of slightly cross-linked networks of homopolymers, can exist in two distinct phases of swollen and collapsed in a liquid. A number of studies on polymer gels have shown discontinuous volume phase transitions in response to external stimuli. ${ }^{2,3}$ In 1984, Hirokawa and Tanaka found that neutral $\operatorname{poly}(N$ isopropylacrylamide) (NIPA) gel, which is weakly crosslinked by $N, N^{\prime}$-methylene-bis-acrylamide (BIS), can exhibit the volume phase transition in water only by changing the temperature. ${ }^{4}$ The transition mechanism was attributed to the change in the balance of hydrophilic and hydrophobic interactions. The discontinuity of the transition is very small, which is related to the fact that the neutral NIPA gel is near the critical point. ${ }^{4,5}$ The effects of the composition of NIPA, BIS, and total monomer concentration on the transition behavior have been well investigated. ${ }^{5}$ The effects of the ionization on the swelling behavior of NIPA gels have also been extensively studied using the weakly ionized NIPA gels, ${ }^{6}$ which are copolymerized by ionizable monomers such as sodium acrylate (SA) and acrylic acid (AA) at gelation. In

\footnotetext{
a) Author to whom all correspondence should be addressed; electronic mail: suzuki@post.me.ynu.ac.jp
}

the case of the ionized NIPA gel, the swelling ratio of the swollen state is increased and the transition temperature is also increased with increasing the amount of ionizable monomers. The qualitative swelling behavior of the ionized NIPA gels has been successfully described by the equation of state of gels on the basis of the Flory-type free energy. ${ }^{2,6,7}$ Although the static swelling behavior as well as the kinetic properties of NIPA gels have been extensively studied, the phase transition velocity and its fundamentals have not been fully understood yet. As for the kinetic properties of polymer gels, most studies ${ }^{8-13}$ have been investigated on the basis of the collective diffusion of polymer networks; the TanakaFillmore model ${ }^{8}$ has often been used to understand the time course of the volume change. In this model, the diameter change to approach an equilibrium state is expressed by a simple function of time after the change in the environment conditions. For the general situations, such as bulk gels with all sorts of shapes, the basic kinetic mechanism has not been well understood, and no theory can explain completely the initial to final size change of the phase transition. We believe that the reason for the deficient model results from failure to consider network imperfections and that this is also a problem related to the network structures. Recently, we have studied the conformation changes during the shrinking phase transition of cylindrical neutral NIPA gels. ${ }^{14}$ In this neutral (nonionized) NIPA gel, the shrinking phase transition starts 
from the surface, showing a fine pattern. The collapsed portion develops from the collapsed surface layer to the core portion in a different manner depending on the temperature drift rate, $\nu_{T}$, or the degree of "super-heating," $\Delta T$. It was found that the phase transition velocity has a strong correlation to the macroscopic conformation changes, which can be determined by the phase diagram of the polymer gels. On the other hand, in the present ionized NIPA gels, the conformation change is expected to occur in a quite different manner. This is because the balance of the osmotic pressure should be totally different from that of the neutral NIPA gel due to the counterions. In fact, it has been well established that the ionized gels would exhibit a bubble pattern ${ }^{12,15-17}$ during the shrinking phase transition, which is restricted in the neutral gels. ${ }^{14}$

In this paper, our interest was focused on the macroscopic conformation change and the kinetic properties of the weakly ionized NIPA gel on the shrinking phase transition. The gel has a long cylindrical shape with submillimeter diameter. ${ }^{18,19}$ The macroscopic conformation changes during the shrinking phase transition are presented in order to establish an underlying foundation of the phase-separation processes. We will discuss the observations during the shrinking phase transition in terms of the conventional model of the nucleation and the spinodal decomposition, and present the mechanism of the phase-separation to make-up the collapsed network.

\section{EXPERIMENTAL METHOD}

Gels were synthesized by a free radical polymerization reaction in a cylindrical shape with submillimeter diameter. Weakly ionized NIPA gels were prepared by exactly the same method as the neutral NIPA gels described in Ref. 14 except for the amount of the main constituent. In the pre-gel solution a small amount $(1.16 \%)$ of total NIPA monomer was replaced by AA. In this synthesis, the base solution is as follows; $7.70 \mathrm{~g}$ of purified NIPA (main constituent, Kohjin), $144 \mathrm{~g}$ of purified AA (ionizable monomer, Wako), $133 \mathrm{mg}$ of BIS (cross-linker, Wako), and $240 \mu \mathrm{l}$ of $N, N, N^{\prime}, N^{\prime}-$ tetramethylethylenediamine (TEMED, acceralator, Wako) were dissolved in $100 \mathrm{~g}$ distilled de-ionized water (pure water), hereafter the solution was stored at the ice temperature. After the solution was fully saturated with nitrogen by bubbling, $40 \mathrm{mg}$ of ammonium persulfate (APS, initiator, Wako) was added to this mixture to initiate the reaction. Hereafter, the glass microcapillaries with an inner diameter of 141.5 $\mu \mathrm{m}$ were inserted into the pregel solution. The gelation was carried out at the ice temperature for at least $12 \mathrm{~h}$. After the gelation had completed, cylindrical gels were removed from microcapillaries and subsequently washed in pure water to wash away residual chemicals as well as unreacted monomers from the polymer networks. In order to destroy the structure formed at gelation by the hydrogen bonding due to the carboxyl groups, the gels were dipped into the aqueous sodium hydroxide $(p \mathrm{H}=14)$, and were successively washed in pure water.

The experimental setup was similar to that used in Ref. 14. Temperature controlled water with an accuracy of $\pm 0.05^{\circ} \mathrm{C}$ was circulated in the cell. After reaching equilib- rium at each temperature, the gel was then imaged by an optical microscope apparatus with calibrated charge-coupled device (CCD) camera and video processors. For the measurement of phase transition velocity, the temperature was continuously increased with a constant heating rate, $\nu_{T}$, using a computer controlled water bath on the continuous heating process. The constant $\nu_{T}$ was variable in the range between $0.00021^{\circ} \mathrm{C} \mathrm{min}^{-1}\left(1{ }^{\circ} \mathrm{C}\right.$ per $\left.80 \mathrm{~h}\right)$ and $1^{\circ} \mathrm{C} \mathrm{min}^{-1}$. On the isothermal process, the circulating initial water temperature was abruptly increased to the final temperatures by switching the water flow from one water bath to another. Note that the several gels were used for all measurements. Therefore, the absolute diameter and the transition temperature depend on the sample. This is because it is very difficult to control the degree of washing by pure water, which could determine the amount of the replaced proton by $\mathrm{Na}$ ions. Nevertheless, one can believe that it will not disturb the general pictures, since the gels were synthesized from the same solution at the same time; the gels exhibit quantitatively the same shrinking behavior within the present macroscopic measurements.

\section{EXPERIMENTAL RESULTS}

\section{A. Swelling curve}

We first measured the diameter of the weakly ionized NIPA gel as a function of temperature. Figure 1 shows the diameter during the continuous heating process with a constant $\nu_{T}, 0.00021{ }^{\circ} \mathrm{C} \mathrm{min}^{-1}$ (the minimum drift rate in our apparatus). On this gradual heating process, the present gel started shrinking at $35.96{ }^{\circ} \mathrm{C}$ from the edge of the cylindrical sample. The starting temperature of the transition depends on samples resulting from the treatment of dipping the gels into the aqueous sodium hydroxide and successively washing it by pure water. The treatment conditions, such as the volume ratio of gel/solvent, the dipping time, the washing time, etc., are not exactly the same for each gel for technical reasons. The gels have different compositions of the solvent, resulting in the different transition temperature. It has been experimentally pointed out that the phase transition of polymer gels begins at the surfaces, which is a consequence of long-range elastic effects. ${ }^{20,21}$ In the case of the cylindrical gels, one can expect that the phase transition begins at the corners of the edges, since the free energy gain by the transition is largest at the corners. It should be noted that the transition can start at the different positions from the edges, including the positions of a large scratch on the surface, or the cave inside the gel. In the present selected samples (with less imperfections), the first collapsed phase emerges at the edges. It develops with increasing the temperature, and the gel falls into the completely collapsed state at $38.96{ }^{\circ} \mathrm{C}$.

One can say that the gel takes a completely swollen state below $35.96{ }^{\circ} \mathrm{C}$, and totally collapsed state above $38.96{ }^{\circ} \mathrm{C}$ on this continuous heating process with $0.00021^{\circ} \mathrm{C} \mathrm{min}^{-1}$. Between both temperatures, the gel exhibits a phase coexistence, ${ }^{22,23}$ although it is not equilibrium (having a long relaxation time, which is much longer than the characteristic relaxation time in this tiny gel ${ }^{18,19}$ ); when $\nu_{T}$ could decrease, the finishing temperature of the transition would decrease, 

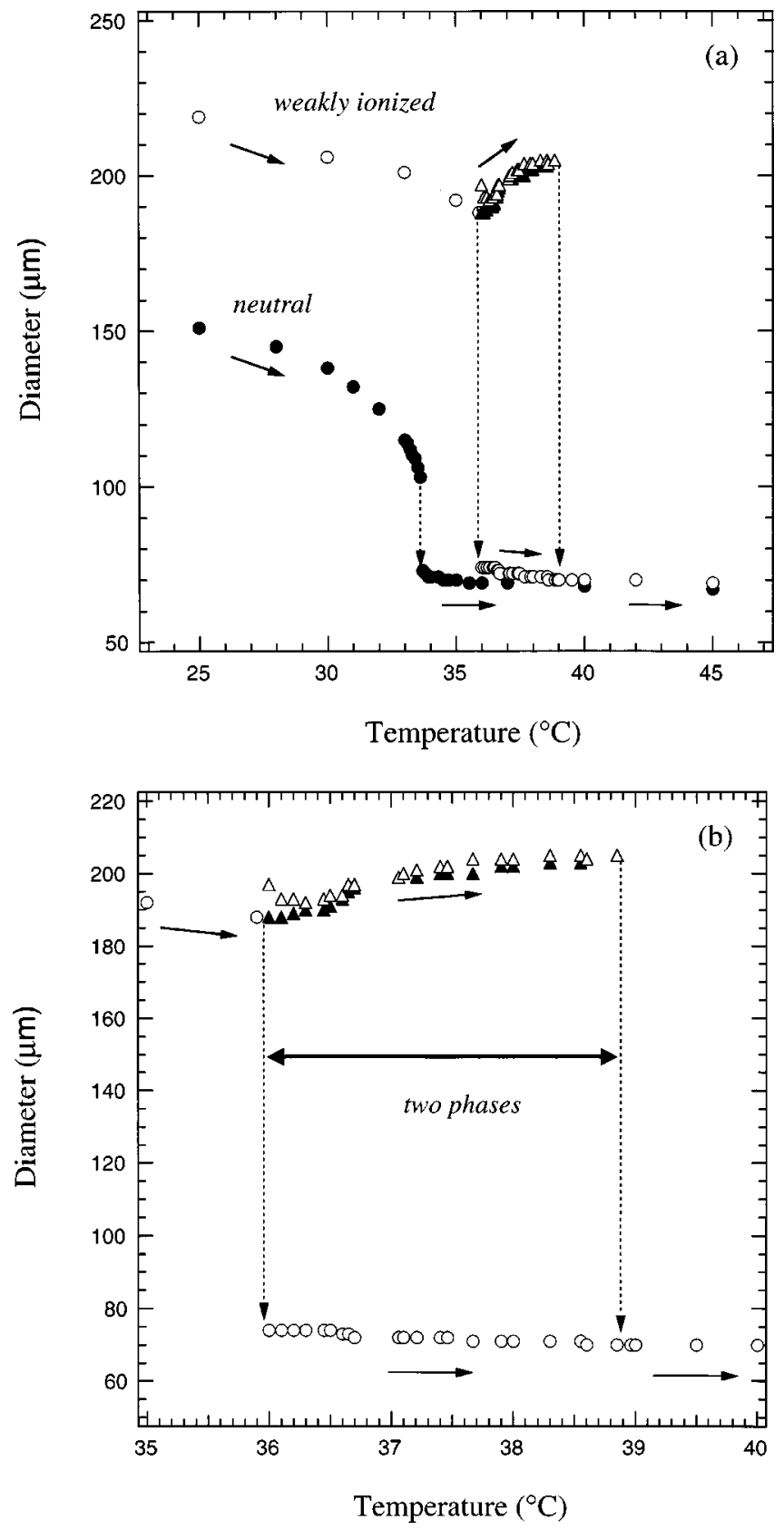

FIG. 1. (a) Diameter change of the weakly ionized NIPA gel (open circles and triangles) as a function of temperature on a continuous heating process with a constant temperature drift rate, $\nu_{T}$, of $0.00021^{\circ} \mathrm{C} \mathrm{min}^{-1}$ (the minimum $\nu_{T}$ used in the present experiment), together with the equilibrium diameter change on heating of the neutral NIPA gel (closed circles). (b) The details of the phase coexistence of the weakly ionized NIPA gel. Between 35.9 and $38.9^{\circ} \mathrm{C}$, the gel can take two phases, depending on the thermal hysteresis. Open triangles denote the swollen diameter at the phase boundary, and the closed triangles indicate the swollen diameter far from the boundary.

but it will not become the starting temperature of the transition at the limit of $\nu_{T} \rightarrow 0$. This corresponds to the fact that the weakly ionized NIPA gel can exhibit the equilibrium phase coexistence in the vicinity of the transition point, ${ }^{20}$ which will be discussed later.

It is interesting to observe that the swollen diameter in the phase coexistence is evidently larger than the diameter just below the transition temperature; the swollen diameter
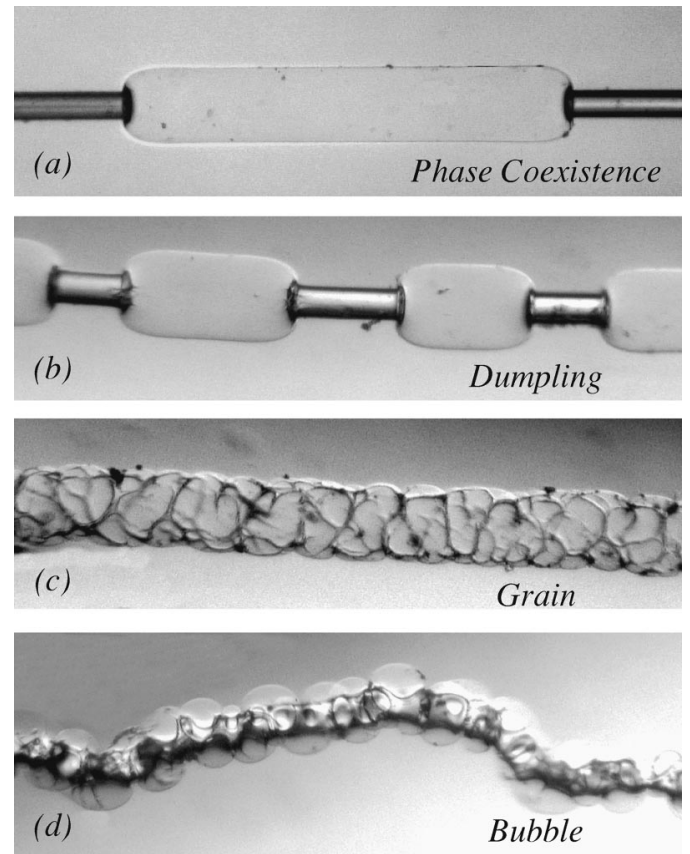

FIG. 2. Characteristic pictures of the shrinking phase transition of the weakly ionized NIPA gel on a continuous heating process. The temperature was increased with a constant temperature drift rate, $\nu_{T}$, in the range between 0.00021 and $1{ }^{\circ} \mathrm{C} \mathrm{min}^{-1}$. The characteristic pictures include the phase coexistence (a), the linked dumplings (b), the grain pattern (c), and the bubble pattern (d). The collapsed diameters are in $70 \pm 2 \mu \mathrm{m}$.

increases slightly but discontinuously, when the initial collapsed portion appears. Note that the swollen diameter in the vicinity of the phase boundary is slightly larger than that far from the boundary.

\section{B. Shrinking conformation change and transition temperature on continuous heating processes}

The conformation change and the phase transition temperature were obtained in the continuous heating processes with the constant $\nu_{T}$ in the range between 0.00021 and $1{ }^{\circ} \mathrm{C} \mathrm{min}^{-1}$ (the maximum drift rate in the present experiment). The conformation change strongly depends on $\nu_{T}$. Several characteristic conformations obtained by the optical microscope are shown in Fig. 2, which are totally different from those of the neutral NIPA gel. ${ }^{14}$ When $\nu_{T}$ is small enough, the phase coexistence can be seen, and the number of the locally collapsed portions (linkages between dumplings) increased one by one with increasing $\nu_{T}$. The shape is like "linked dumplings." When $\nu_{T}$ exceeds a threshold (between 0.0033 and $0.0083^{\circ} \mathrm{C} \mathrm{min}^{-1}$ ), the coarse surface pattern, which looks like a "grain," can be observed on the whole surface at the first stage of the phase transition. Some collapsed patterns develop at the expense of the growth of the other patterns, hereafter the gel takes "linked dumplings." On the other hand, when $\nu_{T}$ is large enough (above $0.033{ }^{\circ} \mathrm{C} \mathrm{min}^{-1}$ ), the grain surface pattern slowly develops (being subdivided) without the linked dumplings, and the bubble pattern appears on the surface. The bubbles shrink, disappear one by one, and the gel finally becomes the completely collapsed state. The details of the conformation 

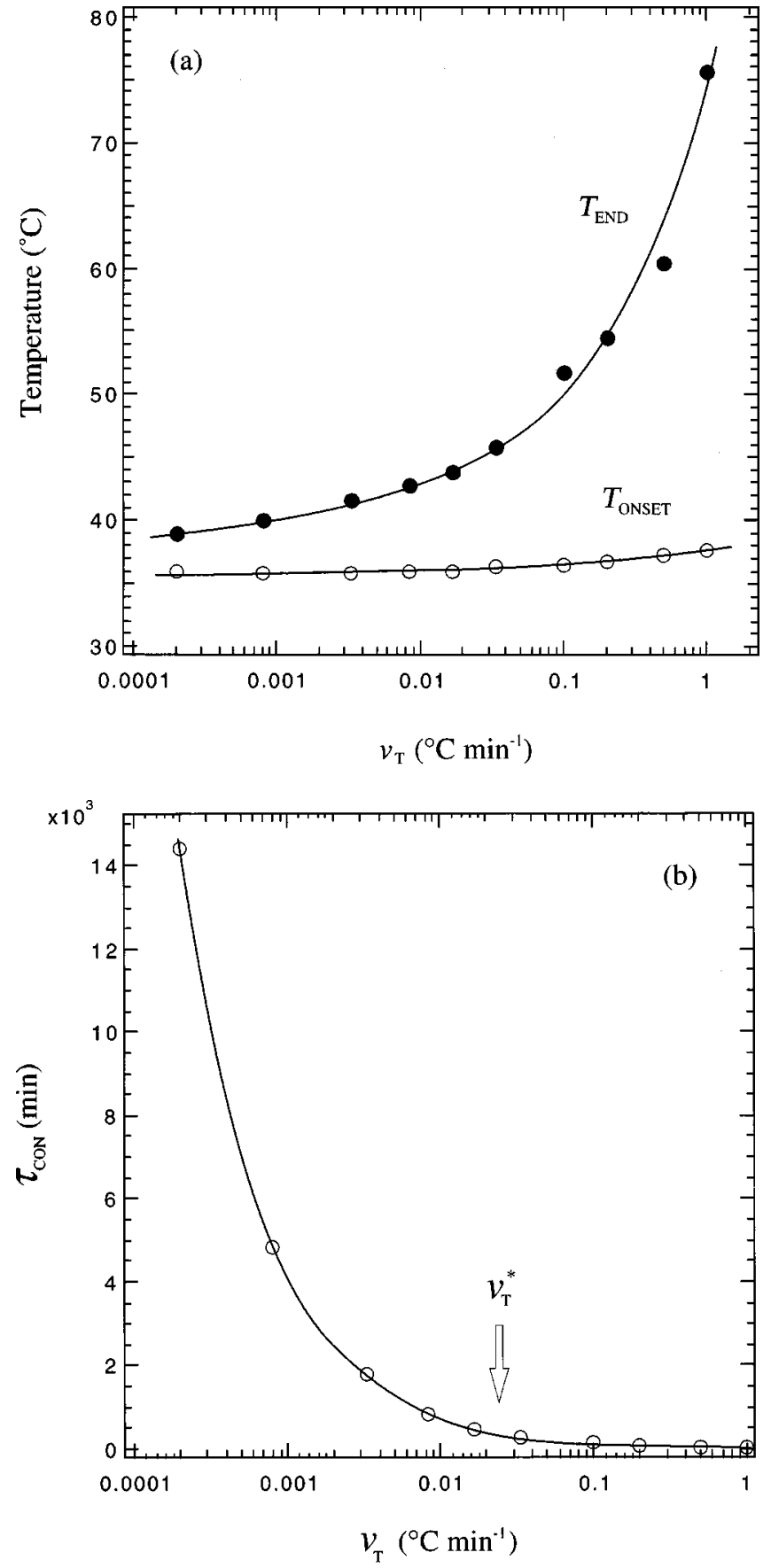

FIG. 3. (a) Characteristic temperatures on the continuous heating processes with constant temperature drift rates, $\nu_{T} ; T_{\text {onset }}$ and $T_{\text {end }}$ in the whole range of $\nu_{T}$ between 0.00021 and $1{ }^{\circ} \mathrm{C} \mathrm{min}^{-1}$. (b) Time, $\tau_{\text {con }}$, from onset to end of the swollen to collapsed phase transition as a function of the temperature drift rate, $\nu_{T}$, on the continuous heating processes. Lines added are guides to the eye. $\nu_{T}^{*}$ indicates the threshold below which the phase coexistence (or linked dumplings) can appear.

change depend on $\nu_{T}$; the larger $\nu_{T}$, the smaller the bubble becomes. Even when $\nu_{T}$ is $1^{\circ} \mathrm{C} \mathrm{min}{ }^{-1}$, the gel remains transparent with a large number of fine surface bubbles, and the surface becomes smooth with time.

Figure 3(a) shows the onset and end temperature of the phase transition; the onset temperature, $T_{\text {onset }}$ is defined as the temperature where the collapsed portion appears at the corner of the edge. The end temperature, $T_{\text {end }}$ is defined as the temperature where the length change stops. One can see that $T_{\text {onset }}$ is almost constant (or slightly increases) with in- creasing $\nu_{T}$, while $T_{\text {end }}$ largely increases. Figure 3(b) shows the time, $\tau_{\text {con }}$, to complete the swollen to collapsed phase transition in the continuous heating process; $\tau_{\text {con }}=\left(T_{\text {end }}\right.$ $\left.-T_{\text {onset }}\right) / \nu_{T}$. One can see that $\tau_{\text {con }}$ rapidly decreases with increasing $\nu_{T}$ in the small $\nu_{T}$ range, and $\tau_{\text {con }}$ becomes small in the larger $\nu_{T}$ range. It should be noted that the characteristic $\nu_{T}^{*}$, marked in Fig. 3, corresponds to the limit of the appearance of the phase coexistence (or linked dumplings), indicating that the dependence of $\tau_{\text {con }}$ on $\nu_{T}$ is also related to the conformation change.

\section{Phase transition velocity on steplike temperature increase}

The characteristic conformation change can be clearly observed in the isothermal process after a steplike temperature increase beyond the transition point, which depends on the degree of "super-heating," $\Delta T$ (the difference between the heating transition temperature and the final temperature). The conformations of the gels obtained by the optical microscope are shown in Fig. 4. The phase coexistence (linked dumplings) [Fig. 4(a)], as well as the shrinking bubble pattern [Fig. $4(\mathrm{~b})]$ in the case of smaller or larger $\Delta T$ corresponds to that of smaller or larger $\nu_{T}$, respectively. In this isothermal process, however, when $\Delta T$ exceeds a threshold (between 11 and $12{ }^{\circ} \mathrm{C}$ ), another characteristic conformation can be seen after the bubble pattern disappears; at the final stage of the transition, the gel becomes opaque, and the transparent surface skin (collapsed phase) develops with time. The opaque core portion becomes smaller and smaller with time, and finally disappears to become a completely collapsed phase, which could not be observed for the smaller $\Delta T$ or on the present continuous heating process.

Figure 5 shows the time, $\tau_{\text {iso }}$, to finish the swollen to collapsed phase transition in the isothermal process after a steplike temperature increase, $\Delta T$. In this measurement, the solvent temperature was quickly increased beyond the transition point from $35.9^{\circ} \mathrm{C}$ (just below the transition point) to the respective final temperature. As previously mentioned, when $\Delta T$ is small enough, $\tau_{\text {iso }}$ is infinite because of the phase coexistence. One can see that $\tau_{\text {iso }}$ decreases rapidly with increasing $\Delta T$, then slightly decreases in the larger $\Delta T$. We have two characteristic super-heating degrees, $\Delta T_{1}^{*}$ and $\Delta T_{2}^{*}$, marked in Fig. 5; below $\Delta T_{1}^{*}$ the phase coexistence (or linked dumplings) appears, while above $\Delta T_{2}^{*}$ the opaque phase appears. The intermediate region between $\Delta T_{1}^{*}$ and $\Delta T_{2}^{*}$ suggests a transformation of two competing mechanisms, characterized by the appearance of the bubble pattern and its disappearance.

The number of the collapsed portions (linkages between dumplings), $N_{C}$, in the linked dumplings increases with $\Delta T$, as is seen in the conformation changes (Fig. 4). Figure 6 shows $N_{C}$ and the time to form the first collapsed portion, $\tau_{C}$, as functions of $\Delta T$. One can see that $N_{C}$ monotonically and rapidly increases with $\Delta T$, while $\tau_{C}$ correspondingly decreases. As will be discussed later, $\tau_{C}$ is related to the formation and the growth of the linkage.

It should be noted that not only the absolute value of the transition temperature but also the swelling ratio is very reproducible on all processes in the same gel. The respective 
$\Delta T=0.5{ }^{\circ} \mathrm{C}$
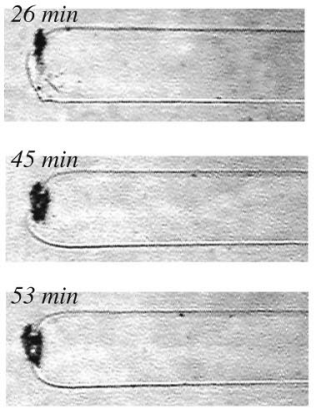

$125 \mathrm{~min}$

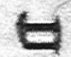

$1135 \mathrm{~min}$

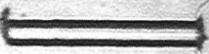

(a)
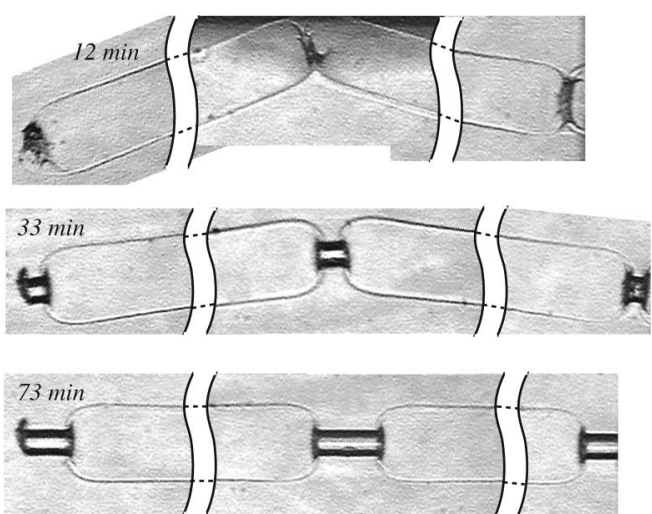

$\Delta T=1.5{ }^{\circ} \mathrm{C}$
$\Delta T=7{ }^{\circ} \mathrm{C}$

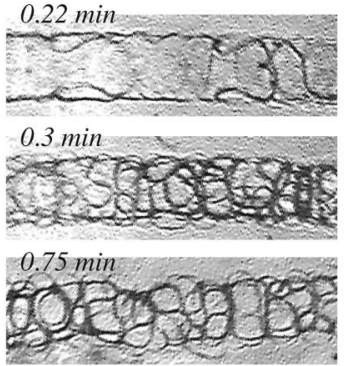

$1.5 \mathrm{~min}$
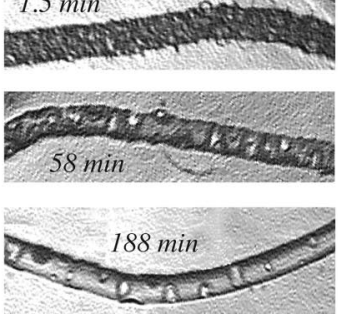

$327 \mathrm{~min}$

(b)
$\Delta T=11{ }^{\circ} \mathrm{C}$
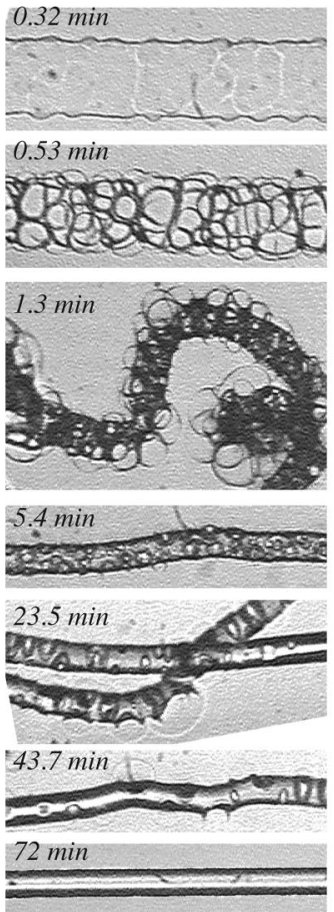
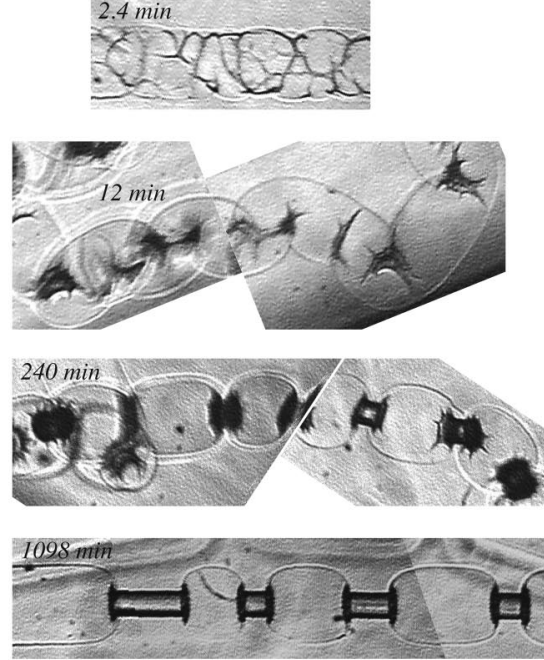

$\Delta T=20{ }^{\circ} \mathrm{C}$
$\Delta T=12{ }^{\circ} \mathrm{C}$

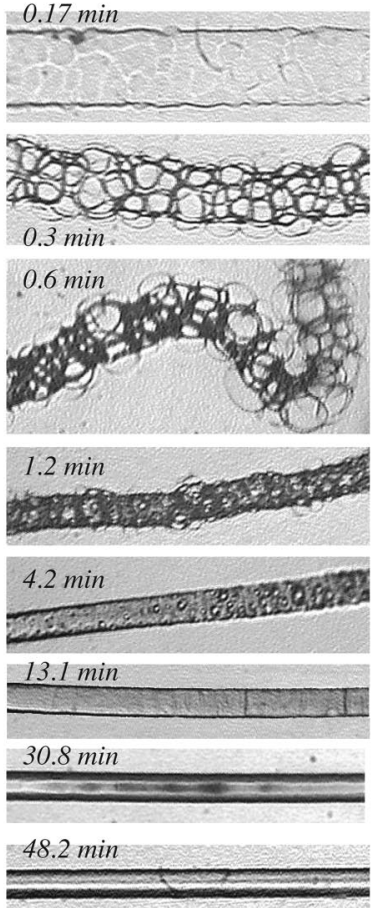

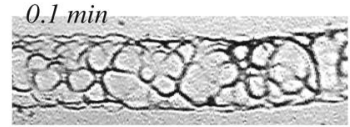
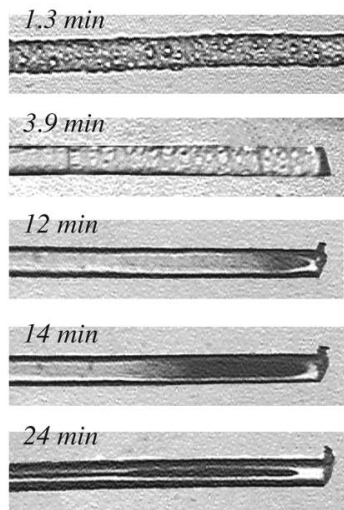

$38 \mathrm{~min}$

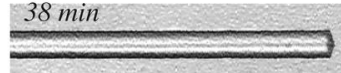

FIG. 4. Pictures of the shrinking phase transition of the weakly ionized NIPA gel on the isothermal processes after the temperature is jumped from $35.9^{\circ} \mathrm{C}$ to several final temperatures; (a) the phase coexistence (or linked dumplings) in the cases of $\Delta T=0.5,1$, and $1.5^{\circ} \mathrm{C}$, (b) the bubble pattern and the opaque phase in the cases of $\Delta T=7,11,12$, and $20^{\circ} \mathrm{C}$. Time evolution of the conformation change depends on $\Delta T$. The collapsed diameters of all processes are in $70 \pm 2 \mu \mathrm{m}$.

value is the same within the present experimental accuracy even after the gel experienced the surface bubbles, as well as even after the gel went back to the initial swollen state from the deepest quenches. Such reversibility does not occur if the stress due to the formation of the surface bubbles induces the permanent plastic deformation in the intermediate size level. ${ }^{17} \mathrm{We}$ believe that the appearance of the surface bubbles might permanently change the chain structure in the molecular level. However, it does not change the network structure in the intermediate size level, such as the micro- domain structure, which could determine the macroscopic elastic properties of the present polymer gels. ${ }^{24}$

\section{DISCUSSION}

\section{A. Characteristic conformations during the shrinking phase transition}

In the present study, several interesting features appeared in the shrinking phase transition of the weakly ionized NIPA gels. First, the macroscopic behavior on both pro- 


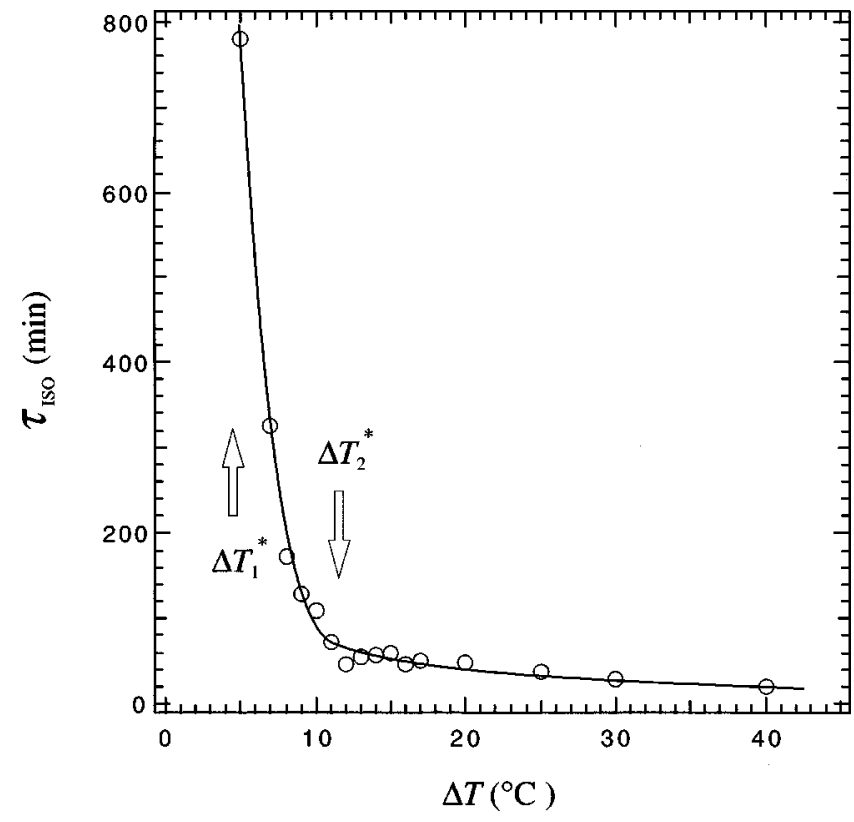

FIG. 5. Time, $\tau_{\text {iso }}$, from onset to end of the swollen to collapsed phase transition as a function of the degree of "super-heating," $\Delta T$ on the isothermal process after several steplike temperature increases beyond the transition point. The initial temperature was $35.9{ }^{\circ} \mathrm{C}$ (just below the transition point). Following a rapid decrease below $\Delta T=12{ }^{\circ} \mathrm{C}$ (final temperature is $\left.47.9^{\circ} \mathrm{C}\right)(\mathrm{a}), \tau_{\text {iso }}$ exhibits a slight decrease (b). Lines added are guides to the eye.

cesses can be characterized by four conformation changes: the phase coexistence (or linked dumplings), the grain pattern, the bubble pattern, and the opaque phase in the core portion. Those have strong correlation with the phase transition velocity against $\nu_{T}$ or $\Delta T$, which are summarized in Fig. 7.

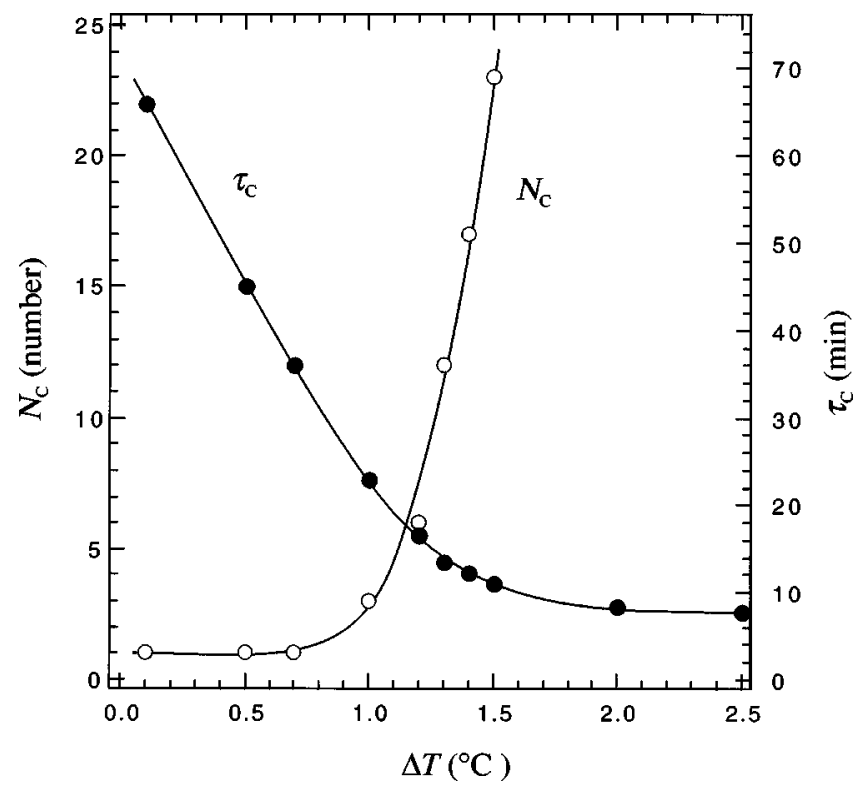

FIG. 6. Number of collapsed portion (linkage between dumplings), $N_{C}$, in the linked dumplings and the time to form the linkage, $\tau_{C}$, as functions of $\Delta T$ on the isothermal processes after the temperature is jumped from $35.9^{\circ} \mathrm{C}$ to several final temperatures. One can see that $N_{C}$ monotonically increases with $\Delta T$, while $\tau_{C}$ correspondingly decreases.
The phase coexistence was observed on both processes under respective conditions, $\nu_{T} \leqslant 0.0033^{\circ} \mathrm{C} \mathrm{min}^{-1}$ or $\Delta T$ $\leqslant 1.4^{\circ} \mathrm{C}$. It is believed that the phase coexistence is one of the most interesting properties of the ionized polymer gels reported in the literature. ${ }^{22,23}$ The neutral NIPA gel does not exhibit it, but transforms from the swollen to collapsed phase by raising the temperature only $0.1^{\circ} \mathrm{C} .{ }^{14}$ The origin of the phase coexistence is an unsolved problem at the present time, which was pointed out in two ionized gels; the ionized poly(acrylamide) gels in response to an external electric field ${ }^{25}$ and the ionized NIPA gels in response to a temperature change. ${ }^{20}$ In the latter example, the breakdown of the Gibbs phase rule is pointed out: ${ }^{23}$ The phase coexistence is attributed to the irregular distortion of networks at the transition by transition-induced internal stress, where the duration of the temperature range of phase coexistence can be enhanced by increasing the ionization. As is shown in Fig. 1, the swollen part tends to swell with temperature even after the phase transition starts. This can be explained by the theoretical prediction, ${ }^{26}$ as well as the experimental observations, ${ }^{27-29}$ where a swollen gel will swell upon stretching. We have been conducting experiments to give an answer to the phenomena, which will be published elsewhere. We believe that not only the network collective diffusion but also the ion diffusion in the solvent should be taken into account.

When $\nu_{T} \geqslant 0.0083^{\circ} \mathrm{C} \mathrm{min}^{-1}$ or $\Delta T \geqslant 1.5^{\circ} \mathrm{C}$, the phase transition progresses in two stages: At the first stage, the grain pattern appears on the entire surface due to the macroscopic phase separation, indicating that the phase transition starts at the surface. At the second stage, the linkeddumplings appear, if $\nu_{T} \leqslant 0.0167^{\circ} \mathrm{C} \mathrm{min}{ }^{-1}$ or $\Delta T \leqslant 4{ }^{\circ} \mathrm{C}$. In the case of the gel system, the phase separation occurs not only between the swollen and the collapsed networks but also between the swollen network and the solvent, since the gel is an open system, ${ }^{23}$ therefore the phase separation by quenching is accompanied by volume shrinkage. The former is a solidlike property (local polymer interaction), and the latter is a liquidlike property (collective polymer diffusion). The initial surface grain pattern is mainly attributed to the results from the former phase separation. On the other hand, when $\nu_{T} \geqslant 0.033{ }^{\circ} \mathrm{C} \mathrm{min}^{-1}$ or $\Delta T \geqslant 5{ }^{\circ} \mathrm{C}$, the bubble pattern appears at the second stage, shrinks with time, and finally disappears (not showing the linked dumplings). The shrinkage of the bubbles results mainly from the latter phase separation. If $\Delta T \geqslant 12{ }^{\circ} \mathrm{C}$ on the isothermal process, the opaque phase appears in the core portion. In this case, the gel shrinks after showing the grain and the bubble patterns, and its surface becomes quickly smooth. The phase-separation mechanism of this phase is totally different from that of the phase coexistence.

The observed characteristic conformation changes should be related to the different mechanisms of phase separation due to the different heating conditions. The most possible explanation lies with the traditional phase-separation phenomena. ${ }^{30,31}$ The macroscopic conformation change during the phase transition in some binary systems can be interpreted in terms of the phase separation of two mechanisms; the nucleation growth (coarsening) and the spinodal 


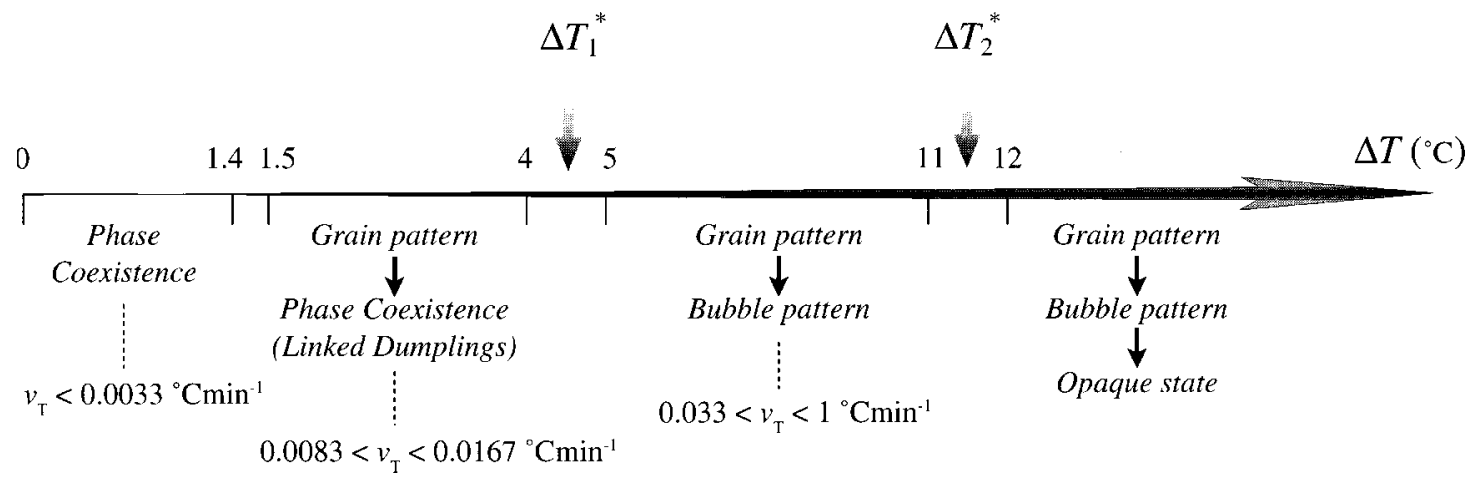

FIG. 7. Summary of the relationship between the conformation change and the heating conditions, classified by $\Delta T$ on the isothermal process and $\nu_{T}$ on the continuous heating processes.

decomposition. $^{31-33}$ On the isothermal process in the present system, the phase coexistence in the small $\Delta T$ range can be dominated by the nucleation mechanism in the metastable region. On the other hand, the opaque phase in the large $\Delta T$ range is dominated by the spinodal decomposition in the unstable region. The opaque phase on the deep quenches can be attributed to the frozen inhomogeneities, ${ }^{34}$ which is a consequence of the vicinity of the Lifshitz point: The microphase separation crosses over to the macrophase separation and the gel exhibits strong density variation on length scales comparable to the wavelength of visible light. In the intermediate $\Delta T$ range, however, the phase transition comes into the crossover (mixed-state) region. The different conformation changes could be explained by the combination of two phase-separation mechanisms. The large intermediate $\Delta T$ region was also reported in the case of the neutral NIPA gels. ${ }^{14}$ However, the phase coexistence (or linked dumplings) and the bubble pattern were observed only in the ionized gels.

It should be noted that the opaque phase could not be observed on the continuous heating process with the larger $\nu_{T}$ region, although $T_{\text {end }}$ exceeds the final temperature where the opaque phase can be observed on the isothermal process $\left(\Delta T \geqslant \Delta T_{2}^{*}\right)$. This is because the phase separation due to the nucleation mechanism is still dominant even at the maximum $\nu_{T}, 1{ }^{\circ} \mathrm{C} \min ^{-1}$ in the present experiment; the phase separation by the spinodal decomposition could not form the macroscopic opaque phase, since the nucleation occurred dominantly in the center core of the cylindrical gel. If we can continuously increase the temperature much faster, the macroscopic opaque phase could be observed before the phase transition is completed.

The characteristic conformation changes results from the same principles in both processes, therefore we will discuss the details of the conformation changes on the isothermal processes in the following sections.

\section{B. Thermally activated nucleation and origin of "linked dumpling"}

The critical difference between the traditional nucleation phenomena and the phase separation in the present system is attributed to the unique structure of polymer gel, that is, a network of weakly cross-linked polymers with multiple free- doms, swollen in a liquid. The network in a polymer gel system is continuously connected to form a dilute threedimensional solid with a complicated structure. Thermally activated nucleation could be strongly suppressed in bulk polymer gels, which is predicted by the theoretical arguments. $^{26,35}$ This is because the embryos (or clusters) of the collapsed phase could not remain in the local potential minimum, since the energy gain from the network volume change will not exceed the loss of the elastic energy from the boundary strain. However, the present study suggests that the nucleation is expected to appear on the surface in the first stage of the transition. Of the various explanations being considered for the appearance of the nucleation, the imperfection of the surface structure is essential; the network surface structure is far from the perfect lattice, but has much more defects than in the core portion. According to the microscopic structure observed by an atomic force microscopy (AFM), the spongelike domains exist on the NIPA gel surfaces. $^{36}$ Moreover, the side chains ${ }^{5,37}$ (short linear NIPA polymers), having the lower transition temperature, ${ }^{38}$ are expected to exist on the surfaces, which could not be observed by the AFM measurement. Nucleation could start with such portions. Therefore, the volume phase transition takes place by means of the nucleation mechanism, when the gel stays between the binodal and the spinodal lines of the swollen state of the gel. According to the theoretical consideration, ${ }^{34}$ it should be noted that the bulk nucleation is expected in quasi-one dimensional gels, even if such a network imperfection could be ignored, which supports the present experimental observations.

Among the characteristic conformation changes, the linked dumplings are the most specific conformation. When the phase transition starts, the nuclei develop by combining with each other, and the initial collapsed portion can emerge on the surface through the nucleation mechanism. If the number of the nuclei is small enough $\left(\Delta T \leqslant 1.4{ }^{\circ} \mathrm{C}\right)$, the most dominant nuclei can develop to become clusters, forming the phase coexistence. When the number of nuclei exceeds a threshold, the clusters can form the grain pattern ( $1.5^{\circ} \mathrm{C} \leqslant \Delta T<\Delta T_{1}^{*}$ ), which finally forms the linked dumplings. If it further increases beyond a threshold $(\Delta T$ $\left.>\Delta T_{1}^{*}\right)$, the grain pattern becomes the bubble pattern. The grain pattern can be observed at the initial stage of the phase 
transition, if $\Delta T>1.5^{\circ} \mathrm{C}$. We believe that just after the surface temperature exceeds $35.9^{\circ} \mathrm{C}(\Delta T=0)$, the embryos on the surface are able to become nuclei of the new collapsed phase, forming the grain pattern, and the coarsening of nuclei increases the collapsed volume from the outside to the core portion.

The nucleation mechanism is characterized by two factors of the formation rate as well as the coarsening velocity of the nuclei, which is discussed in Ref. 14. In the classical nucleation model, ${ }^{30,33}$ the formation and the coarsening rates of nuclei depend on the degree of "supercooling" (how deeply the gel state can be brought into the metastable region; in the present case, $\Delta T$ ). The formation rate (or probability) of nuclei is assumed to be proportional to $\exp \left(-G_{V} / k_{B} T\right)$, where $G_{V}$ is the minimum formation energy of nuclei and $k_{B}$ is the Boltzmann constant. This formula is proportional to $\exp \left(-C / \Delta T^{2}\right)$, where the coefficient $C$ is positive, therefore the formation rate rapidly increases with increasing $\Delta T$. On the other hand, the coarsening of nuclei is expected to be a thermal activation process; therefore the coarsening velocity could be expressed in the form of $\exp \left(-G_{C} / k_{B} T\right)$, where $G_{C}$ is the activation energy. This expression also increases with increasing $T$ (that is $\Delta T$ ), since $G_{C}$ does not depend on $T$.

From these expressions, the time evolution of the macroscopic behavior in the metastable region can be well understood, which depends significantly on the absolute value (smaller and larger) of $\Delta T$. As is shown in Fig. 7, when $\Delta T$ exceeds a threshold $\left(\Delta T_{1}^{*}\right)$, the linked dumplings could not be observed, since the coarsening velocity of the nuclei is relatively much larger than the formation rate, where $\tau_{\text {iso }}$ monotonically decreases with $\Delta T$. The number of nuclei and the coarsening velocity will both increase with increasing $\Delta T$, which can accelerate the conformation change at the phase transition, resulting in the decrement of $\tau_{\text {iso }}$ with $\Delta T$. As for the gradual decrement of $\tau_{\text {iso }}$ in $\Delta T>\Delta T_{2}^{*}$, the relaxation of the phase-separated state by the spinodal decomposition turns into the collapsed state with small time constant (same order of the usual collective diffusion constant $^{18,19}$ ), where this behavior could be attributed to the increment of the diffusion constant of polymer networks with $\Delta T .^{39}$ It should be noted that the time constant of the nucleation process in the smaller $\Delta T$ region is much larger than that of the neutral NIPA gel. ${ }^{14}$ The extremely large time constants in this ionized system are attributed to the network properties, as well as the diffusion process of water molecules; the former is that the relaxation during the coarsening process of nuclei requires cooperative motions of the phase boundary at the interface between the collapsed and the swollen portions. Therefore, the neighboring domains at the interface are under mechanical constraint, ${ }^{40}$ which is known to be significantly enhanced by the ionization. ${ }^{29}$ The latter is that the surface layer delays the diffusion of water molecules. The swelling ratio is strongly increased by the small amount of ionization, ${ }^{6}$ therefore the initial network density could be also related to the large time constant.

It is also possible to understand the evidence of the relations between $N_{C}$ and $\Delta T$, as well as $\tau_{C}$ and $\Delta T$. As is shown in Fig. 6, $N_{C}$ monotonically increases with increasing
$\Delta T$, while $\tau_{C}$ correspondingly decreases. From the abovementioned considerations, the nucleation rate and its growth process are enhanced by increasing $\Delta T$, which increases the possibility of the formation of the local collapsed portions, resulting in the increment of $N_{C}$. The coarsening is accelerated by increasing $\Delta T$ due to the increment of the network diffusion constant, resulting in the decrement of $\tau_{C}$. The maximum $N_{C}$ should depend on the total length of the gel, and can be roughly estimated by assuming that the minimum length along the uniaxial direction of the dumpling and the linkage are the same as the swollen or the collapsed diameters, respectively. The total length of the present sample is $20900 \mu \mathrm{m}$, the swollen diameter is about $200 \mu \mathrm{m}$, and the collapsed diameter is about $70 \mu \mathrm{m}$, therefore we obtain roughly the maximum $N_{C}=50$.

\section{Origin of characteristic conformations in the crossover of two mechanisms (grain and bubble patterns and opaque phase)}

When $\Delta T>\Delta T_{1}^{*}$, the bubble pattern appears on the surface at the second stage of the phase transition, instead of the linked dumplings. This is related to the transformation of the phase-separation mechanism from the nucleation to the spinodal decomposition. The problem is what is the limit of the metastable region with increasing $\Delta T$. According to the classical model, ${ }^{31-33}$ one might expect that the transformation from the nucleation to the spinodal decomposition mechanism would take place, in general, at a critical temperature (the spinodal limit should be sharp ${ }^{41}$ ). However, the present experimental observations are not necessarily consistent with this prediction; as mentioned in Sec. III, a crossover (or mixed state) phenomenon of two competing shrinking mechanisms can exist in the characteristic conformation change. Evidence of the existence of crossover cannot be explained by the simple phase diagram. ${ }^{14,23}$ Of the various possibilities to explain the discrepancy, the stress-induced modification of the swollen state should be taken into account in the vicinity of the interface. The present observation of the conformation changes indicates that the shrinking phase transition develops from the surface to the core portion. The surface phase at the initial stage of the shrinking phase transition will minimize the elastic deformation introduced by the nucleation. This is also consistent with the theoretical predictions of the possible nucleation in quasi-onedimensional gels, ${ }^{34}$ as well as the anisotropic surface phase ${ }^{35}$ that is a transient precursor of the bulk phase separation even in the case of the deep quenches.

The appearance of the collapsed networks on the surface can make the elastic distortion in the rest-swollen networks. When the temperature exceeds the metastable limit, the coexistence of the collapsed (skin) and swollen (core) states plays an important role, realized by the anisotropic surface phase in the present long cylindrical gels. It is, therefore, necessary to take into account that the locally collapsed skin layer is subject to stress by proximity to the swollen portion, which is a maximum at the boundary between the two phases. According to the phase transition in mechanically constrained NIPA gels, ${ }^{27-29,40}$ it has been well established that the mechanical stretching induces the increment of the 
transition temperature, as well as the swollen diameter; the collapsed state just above the transition point can return to the swollen state upon application of a small tensile stress. Such an effect on the ionized NIPA gel is much larger than that of the neutral NIPA gel. ${ }^{29}$ When the temperature far exceeds the metastable limit $\left(\Delta T \geqslant \Delta T_{2}^{*}\right)$, these effects can be ignored since the collapsed skin layer will become too thin to affect the relaxation process of the phase-separated network by the spinodal decomposition.

It should be noted that the network structure of the present kind of polymer gels is not homogeneous by nature, but rather consists of microdomains, ${ }^{24,42-45}$ resulting in the inhomogeneous strain distribution at the microscopic level. The hierarchical network structure observed in the neutral NIPA gel ${ }^{44,45}$ affects the phase transition velocity. In order to verify these considerations, it is important to test for the bulk gels with all sorts of shapes. Moreover, not only macroscopic observations but also kinetic experiments at the microscopic level in the same sample are desirable. These are subjects for future investigations.

\section{CONCLUSION}

We have investigated the conformation changes during the shrinking phase transition of the weakly ionized $\operatorname{poly}(\mathrm{N}$ isopropylacrylamide) gels. The experimental results can be summarized as follows. All gels start to shrink from the surface, and the collapsed portion develops from the collapsed surface layer to the core portion in a different manner. Several characteristic patterns, that is, the phase coexistence (or linked dumplings), the grain pattern, the bubble pattern, and the opaque phase, were observed, depending on the temperature drift rate, $\nu_{T}$, or the degree of "super-heating," $\Delta T$. If $\nu_{T}$ or $\Delta T$ is small enough, the phase transition is governed by the nucleation mechanism. When it is large enough, the spinodal decomposition has an important role. The intermediate region suggests a transformation of two competing mechanisms, characterized by the appearance of the bubble pattern and its disappearance. The phase transition velocity has a strong correlation to the surface patterns, which can be determined by the heating conditions. The present results have been discussed qualitatively in terms of the traditional phase-separation model based on the nucleation and the spinodal decomposition, and the inhomogeneous stress distribution during phase separation. The origin of the characteristic conformations is related to the formation rate and the coarsening velocity of the nuclei.

We believe that the results of the present study are the identical properties for the weakly ionized polymer gels with submillimeter diameter; if the gel is neutral, the phase coexistence cannot be observed; if the diameter is comparable to the skin layer, we cannot observe the bubble pattern and the opaque phase. Further experiments, including those for different shapes of gels and for strongly ionized gels, are desirable.

\section{ACKNOWLEDGMENTS}

This work was supported by a Grant-in-Aid for Scientific Research (C), No. 09640684 from the Ministry of Edu- cation, Science, Sports and Culture, by a Grant from Nippon Steel Corporation, and by a Grant from Micromachine Center.

${ }^{1}$ See, for instance, The Polymer Networks Group Series, edited by K. te Nijenhuis and W. J. Mijs (Wiley, Chichester, 1998), Vol. 1.

${ }^{2}$ K. Dusek and D. Patterson, J. Polym. Sci., Part B: Polym. Lett. 6, 1209 (1968).

${ }^{3}$ T. Tanaka, Phys. Rev. Lett. 40, 820 (1978).

${ }^{4}$ Y. Hirokawa and T. Tanaka, J. Chem. Phys. 81, 6379 (1984).

${ }^{5}$ Y. Li and T. Tanaka, Annu. Rev. Mater. Sci. 22, 243 (1992).

${ }^{6}$ S. Hirotsu, Y. Hirokawa, and T. Tanaka, J. Chem. Phys. 87, 1392 (1987).

${ }^{7}$ P. J. Flory, Principles of Polymer Chemistry (Cornell University Press, Ithaca, NY, 1953).

${ }^{8}$ T. Tanaka and D. J. Fillmore, J. Chem. Phys. 59, 5151 (1979).

${ }^{9}$ A. Peters and S. J. Candau, Macromolecules 19, 1952 (1986).

${ }^{10}$ P. Chiarelli and D. De Rossi, Prog. Colloid Polym. Sci. 78, 4 (1988).

${ }^{11}$ T. Tanaka, Physica A 140, 261 (1986).

${ }^{12}$ E. S. Matsuo and T. Tanaka, J. Chem. Phys. 89, 1695 (1988).

${ }^{13}$ Y. Li and T. Tanaka, J. Chem. Phys. 92, 1365 (1990).

${ }^{14}$ A. Suzuki, S. Yoshikawa, and G. Bai, J. Chem. Phys. 111, 360 (1999).

${ }^{15}$ T. Tanaka, S.-T. Sun, Y. Hirokawa, S. Katayama, J. Kucera, Y. Hirose, and T. Amiya, Nature (London) 325, 796 (1987).

${ }^{16}$ E. S. Matsuo and T. Tanaka, Nature (London) 358, 482 (1992).

${ }^{17}$ M. Tokita, S. Suzuki, K. Miyamoto, and T. Komai, J. Phys. Soc. Jpn. 68, 330 (1999).

${ }^{18}$ A. Suzuki and T. Tanaka, Nature (London) 346, 345 (1990).

${ }^{19}$ A. Suzuki, Adv. Polym. Sci. 110, 199 (1993).

${ }^{20}$ S. Hirotsu, J. Chem. Phys. 88, 427 (1988).

${ }^{21}$ E. S. Matsuo and T. Tanaka, J. Chem. Phys. 89, 1695 (1988).

${ }^{22}$ S. Hirotsu, Adv. Polym. Sci. 110, 1 (1993).

${ }^{23}$ S. Hirotsu, Phase Transit. 47, 183 (1994).

${ }^{24}$ A. Suzuki, T. Ejima, Y. Kobiki, and H. Suzuki, Langmuir 13, 7039 (1997).

${ }^{25}$ T. Tanaka, I. Nishio, S.-T. Sun, and S. Ueno-Nishio, Science 218, 467 (1982).

${ }^{26}$ P. Pekarski, A. Tkachenko, and Y. Rabin, Macromolecules 27, 7192 (1994).

${ }^{27}$ S. Hirotsu and A. Onuki, J. Phys. Soc. Jpn. 58, 1508 (1989).

${ }^{28}$ A. Suzuki and S. Kojima, J. Chem. Phys. 101, 10003 (1994).

${ }^{29}$ A. Suzuki, K. Sanda, and Y. Omori, J. Chem. Phys. 107, 5179 (1997).

${ }^{30}$ L. D. Landau and E. M. Lifshitz, Statistical Physics (Pergamon, London, 1962).

${ }^{31}$ P.-G. de Gennes, Scaling Concepts in Polymer Physics (Cornell University Press, Ithaca, 1979).

${ }^{32} \mathrm{~W}$. Gebhardt and U. Krey, Phase Transition and Critical Phenomena (Vieweg \& Sohn, Braunschweig, Wiesbaden, 1980).

${ }^{33}$ D. V. Ragone, Thermodynamics of Materials (Wiley, New York, 1995).

${ }^{34}$ Y. Rabin and S. Panyukov, Macromolecules 30, 301 (1997).

${ }^{35}$ S. Panyukov and Y. Rabin, Macromolecules 29, 8530 (1996).

${ }^{36}$ A. Suzuki, M. Yamazaki, and Y. Kobiki, J. Chem. Phys. 104, 1751 (1996).

${ }^{37}$ S. J. Candau, C. Y. Young, T. Tanaka, L. Lemarechal, and J. Bastide, J. Chem. Phys. 70, 4694 (1979).

${ }^{38}$ M. Heskins and J. E. Guillet, J. Macromol. Sci. Chem. 2, 1441 (1968).

${ }^{39}$ T. Tanaka, E. Sato, Y. Hirokawa, S. Hirotsu, and J. Peetermans, Phys. Rev. Lett. 55, 2455 (1985).

${ }^{40}$ A. Suzuki and T. Ishii, J. Chem. Phys. 110, 2289 (1999).

${ }^{41}$ T. Tanaka, L. O. Hocker, and G. Benedek, J. Chem. Phys. 59, 5151 (1973).

${ }^{42}$ E. S. Matsuo, M. Orkisz, S.-T. Sun, Y. Li, and T. Tanaka, Macromolecules 27, 6791 (1994).

${ }^{43}$ Y. Suzuki, K. Nozaki, T. Yamamaoto, K. Ito, and I. Nishio, J. Chem. Phys. 97, 3808 (1992).

${ }^{44}$ A. Suzuki, M. Yamazaki, Y. Kobiki, and H. Suzuki, Macromolecules 30, 2350 (1997).

${ }^{45}$ Y. Hirokawa, Part 4 Abstracts of Presentations of ERATO Symposia '97, Tokyo, December 1997, p. 37. 